This item was submitted to Loughborough's Research Repository by the author.

Items in Figshare are protected by copyright, with all rights reserved, unless otherwise indicated.

\title{
Pricing based interference control in reversed time division duplex heterogeneous networks
}

PLEASE CITE THE PUBLISHED VERSION

http://dx.doi.org/10.1109/ICC.2016.7510740

PUBLISHER

(C) IEEE

VERSION

AM (Accepted Manuscript)

LICENCE

CC BY-NC-ND 4.0

\section{REPOSITORY RECORD}

Liu, Ye, Sangarapillai Lambotharan, Arumugam Nallanathan, and Kai-Kit Wong. 2019. "Pricing Based Interference Control in Reversed Time Division Duplex Heterogeneous Networks". figshare. https://hdl.handle.net/2134/23768. 


\title{
Pricing Based Interference Control in Reversed Time Division Duplex Heterogeneous Networks
}

\author{
Ye Liu ${ }^{\ddagger}$, Sangarapillai Lambotharan ${ }^{\ddagger}$, Arumugam Nallanathan ${ }^{\S}$, and Kai-Kit Wong ${ }^{\text {W }}$ \\ ${ }^{\ddagger}$ Loughborough University, Loughborough, LE11 3TU, United Kingdom \\ §King's College London, London, WC2R 2LS, United Kingdom \\ 'University College London, London, WC1E 6BT, United Kingdom \\ Email: \{y.liu6, s.lambotharan\}@lboro.ac.uk ${ }^{\ddagger}$, arumugam.nallanathan@kcl.ac.uk ${ }^{\S}$, and kai-kit.wong@ucl.ac.uk
}

\begin{abstract}
We investigate a pricing based approach to control interference from a tier-2 base station (BS) to a tier-1 BS in reversed time division duplex (TDD) multi-antenna systems. The tier-2 BS is being charged for causing interference to the tier-1 BS. Also, the tier-2 BS has to satisfy the signal-tointerference-plus-noise ratio (SINR) targets of its downlink users under a maximum transmission power constraint. Analytical and simulation studies are carried out to understand the behavior of the tier-2 BS for different charges and for different power budgets. Observations from the analyses suggest that the tier-1 BS can perform interference control and/or profit maximization without knowing the downlink channels of the tier-2 BS.
\end{abstract}

\section{INTRODUCTION}

Heterogeneous networks (HetNets) and massive multipleinput multiple-output (MIMO) have been identified as the enabling technologies for the next generation of cellular networks $[1,2]$. To avoid the heavy signalling on training the channels between the tier-1 base stations (BSs) and the tier-2 BSs in conventional time division duplex (TDD) systems, reversed TDD protocol has been proposed in [3], where the tier-2 BSs perform downlink transmissions while the tier-1 users perform uplink transmissions. On the other hand, it is expensive or even infeasible to establish wired backhaul links from the tier-2 BSs (the smaller BSs) to the tier-1 BSs as cellular networks become more densified, so that wireless backhaul has to be considered in certain scenarios [4].

In order to perform interference control from a tier-2 BS to a tier-1 BS while keeping the signalling across the two tiers low in reversed TDD systems, we consider the downlink beamforming problem for the tier-2 $\mathrm{BS}$, where the tier-1 BS charges the tier-2 BS for causing interference. We perform both theoretical analysis and simulation studies on how the tier-2 BS behaves under different charges and different power budgets. Based on the observations from our studies, we believe that the tier-1 BS can perform interference control by probing through a number of charge values, thus removing the need for the tier-1 BS to know the downlink channels of the tier-2 BS as in centralized designs. The same mechanism can also be used in scenarios where the tier-1 BS is interested in making profit from the tier-2 BS, where the profit comes from charging on the interference.

This work has been supported by the Engineering and Physical Science Research Council of the UK, EPSRC, under the grant EP/M015475.
The rest of the paper is organized as follows. Section II presents the system model. Section III describes the downlink beamforming problem of the tier-2 BS and presents a solution to the problem. Section IV and V provide theoretical analyses when the power budget of the tier-2 BS changes and when the charge on interference changes, respectively. Section VI performs simulation studies. Section VII discusses more on the observations and possible applications of the charging-based approach. Finally, conclusions are drawn in Section VIII.

\section{A. Notation}

We use bold lower-case letters and bold upper-case letters to denote column vectors and matrices, respectively. $\mathbf{a}^{\dagger}$ gives the Hermitian of the vector $\mathbf{a}$, and $\mathbf{a}^{T}$ means the transpose of a. $\mathbf{A}(i, j)$ gives the element located on the $i$-th row and $j$-th column of matrix $\mathbf{A}$, and $\mathbf{A} \succeq 0$ means the matrix $\mathbf{A}$ is positive semi-definite. The identity matrix is denoted as $\mathbf{I}$. Also, we use calligraphic upper-case letters to denote sets, e.g., set $\mathcal{A} .(\cdot)^{*}$ indicates the optimal value of the variable.

\section{SySTEM MODEL}

Suppose there is a tier-2 BS serving $K$ single-antenna users in the downlink. Also, suppose there is one tier-1 BS who is located near the tier-2 BS, and the tier-1 BS is serving its $M$ single-antenna users on its uplink channel. The frequency band used for the downlink transmissions of the tier-2 BS is the same as that for the uplink transmissions of the tier-1 BS, so that the downlink transmissions in tier-2 interfere the uplink transmissions in tier-1. The tier-1 BS and the tier-2 BS are equipped with $N^{\text {Tier-1 }}$ and $N^{\text {Tier-2 }}$ antennas, respectively. Denote $\mathcal{K}$ as the set of the downlink users attached to the tier2 BS. Without loss of generality we let $\mathcal{K}=\{1,2, \ldots, K\}$. Let $\mathbf{x}^{(n)}$ be the symbol vector transmitted by the tier-2 BS at a particular instance $n$, which can be written as

$$
\mathbf{x}^{(n)}=\sum_{\forall k \in \mathcal{K}}\left(\mathbf{w}_{k} x_{k}^{(n)}\right)
$$

where $\mathbf{w}_{k}$ is the beamformer applied for user $k$ at the tier-2 BS and $x_{k}^{(n)}$ is the data symbol of user $k$ at instance $n$. The power of $x_{k}^{(n)}$ is assumed to be one since it can be included in $\mathbf{w}_{k}$. All channels are assumed to be quasi-static and we omit the time indices for the beamformers and the channel gains, 
TABLE I

SUMMARY OF NOTATION

\begin{tabular}{|c|c|}
\hline Notation & Definition \\
\hline$c$ & Per-unit charge on the interference caused by the tier-2 BS \\
\hline$K$ & Number of the tier-2 downlink users \\
\hline $\mathcal{K}$ & The set of the tier-2 downlink users \\
\hline$M$ & Number of the tier-1 uplink users \\
\hline $\mathcal{M}$ & The set of the tier-1 uplink users \\
\hline$N^{\text {Tier-1 }}$ & The number of antennas equipped on the tier-1 BS \\
\hline$N^{\text {Tier-2 }}$ & The number of antennas equipped on the tier-2 BS \\
\hline $\mathbf{h}_{k}$ & Channel between tier-2 user $k$ and the tier-2 BS \\
\hline$Q_{m}$ & The transmission power of the $m$-th tier-1 user \\
\hline$\gamma_{k}$ & SINR target of the $k$-th downlink user of the tier-2 BS \\
\hline $\boldsymbol{\vartheta}_{m, k}$ & Channel between tier-1 user $m$ and tier-2 user $k$ \\
\hline$\Theta$ & Channel between the tier-2 BS and the tier-1 BS \\
\hline$\sigma_{k}^{2}$ & The AWGN power at the $k$-th tier-2 user \\
\hline$\varsigma_{k}$ & $\begin{array}{c}\text { Sum of interference and noise power at the } k \text {-th user of the } \\
\text { tier-2 BS generated by users of the tier-1 BS and AWGN }\end{array}$ \\
\hline
\end{tabular}

because the power allocation within the coherence time can be fixed. Also, it is assumed that user $k$ has a minimum signalto-interference-plus-noise ratio (SINR) requirement which has the value of $\gamma_{k}$. Denote $\mathbf{h}_{k}$ as the channel between the tier-2 $\mathrm{BS}$ and its $k$-th user.

Denote $\mathcal{M}$ as the set of the uplink users of the tier-1 BS, and denote the channel between the $m$-th uplink user of the tier-1 BS and the $k$-th downlink user of the tier-2 BS as $\boldsymbol{\vartheta}_{m, k}$. The received signal at the $k$-th downlink user of the tier-2 BS at instance $n$ is given as:

$$
y_{k}^{(n)}=\mathbf{h}_{k}^{T} \mathbf{x}^{(n)}+\sum_{m \in \mathcal{M}}\left(\boldsymbol{\vartheta}_{m, k} \chi_{m}^{(n)}\right)+z_{k}^{(n)},
$$

where $z_{k}^{(n)}$ is the additive white Gaussian noise (AWGN) at user $k$ the variance of which is denoted as $\sigma_{k}^{2}$ and $\chi_{m}^{(n)}$ is the symbol transmitted by the $m$-th user of the tier-1 BS. Let

$$
Q_{m} \triangleq\left|\chi_{m}^{(n)}\right|^{2}
$$

be the transmission power of the $m$-th uplink user of the tier-1 BS, where the power is assumed to be fixed within the coherence time of the channels. Assume $x_{k_{1}}^{(n)}$ and $z_{k_{2}}^{(n)}$ are independent for all $k_{1}$ and $k_{2}$, and $x_{k_{1}}^{(n)}$ and $x_{k_{2}}^{(n)}$ are independent for all $k_{1} \neq k_{2}$. The SINR at the $k$-th downlink user of the tier-2 BS is given as:

$$
\operatorname{SINR}_{k}^{\text {Tier-2 }}=\frac{\left|\mathbf{w}_{k}^{\dagger} \mathbf{h}_{k}\right|^{2}}{\sum_{j \in \mathcal{K} \backslash\{k\}}\left|\mathbf{w}_{j}^{\dagger} \mathbf{h}_{k}\right|^{2}+\varsigma_{k}},
$$

where $\varsigma_{k} \triangleq \sum_{m \in \mathcal{M}}\left(Q_{m}\left\|\boldsymbol{\vartheta}_{m, k}\right\|^{2}\right)+\sigma_{k}^{2}$ is the sum of the interference from the uplink users of the tier-1 BS and the AWGN power of at the $k$-th user terminal. Table I summaries the definitions of the notation.

\section{TIER-2 BS DOWNLINK BEAMFORMING}

Denote the MIMO channel between the tier-1 BS and the

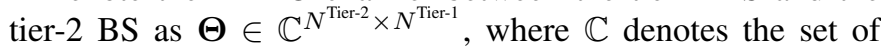
complex numbers. The tier-1 BS sets a nonnegative per-unit charge $c$ on the interference caused by the tier-2 BS. The tier2 BS aims at satisfying the SINR targets of its attached users while minimizing its cost, where the cost of the tier-2 BS is the sum of the transmission power and the price it needs to pay to the tier-1 BS for causing interference. Moreover, the tier-2 BS has a maximum transmission power denoted as $P_{\max }$. We have the following optimization problem for the tier-2 BS:

\section{DOWNLINKBEAMFORMING.}

$$
\begin{aligned}
\operatorname{minimize} & \sum_{\forall k \in \mathcal{K}}\left\|\mathbf{w}_{k}\right\|^{2}+c \cdot \sum_{\forall k \in \mathcal{K}}\left\|\mathbf{w}_{k}^{\dagger} \boldsymbol{\Theta}\right\|^{2}, \\
\text { subject to } & \frac{\left|\mathbf{w}_{k}^{\dagger} \mathbf{h}_{k}\right|^{2}}{\sum_{j \neq k}\left|\mathbf{w}_{j}^{\dagger} \mathbf{h}_{k}\right|^{2}+\varsigma_{k}} \geq \gamma_{k}, \forall k \in \mathcal{K}, \\
& \sum_{\forall k \in \mathcal{K}}\left\|\mathbf{w}_{k}\right\|^{2} \leq P_{\max } .
\end{aligned}
$$

Note that we assume the tier-2 BS knows $\boldsymbol{\Theta}$ and $\left\{\mathbf{h}_{k} \mid k \in\right.$ $\mathcal{K}\}$. Also, the charge set by the tier-1 BS can be an actual monetary charge or a virtual payment mechanism which serves the purpose of interference control. Intuitively, we expect that the larger the value of $c$, the smaller the interference would be caused by the tier- 2 BS towards the tier-1 BS. In case the tier-1 BS tries to make profit by charging the tier-2 BS, which may arise in application scenarios where the tier-1 BS subleases its uplink frequency to the tier-2 BS for downlink transmissions, the way of setting $c$ will also become an important issue. More careful analyses will be given for different values of $c$ later.

We now focus on how to solve DownLINKBEAMFORMING. Our approach is similar to the one in [5], where we examine the Lagrangian dual problem of DowNLINKBEAMFORMING, determine its equivalence to an uplink beamforming problem, and then solve DownLINKBEAMFORMING by solving the equivalent uplink beamforming problem. The applicability of the uplink-downlink duality approach relies on the strong duality of DOWNLINKBEAMFORMING.

Theorem 1. The problem DownlinkBEAmforming is strictly convex and it satisfies strong duality.

Proof. It is shown in [5] that (4) can be written as a second-order cone constraint which is convex. Also, (5) is a summation of norms which defines a convex set. The objective function $\sum_{\forall k \in \mathcal{K}}\left\|\mathbf{w}_{k}\right\|^{2}+c \cdot \sum_{\forall k \in \mathcal{K}}\left\|\mathbf{w}_{k}^{\dagger} \boldsymbol{\Theta}\right\|^{2}=$ $\sum_{\forall k \in \mathcal{K}}\left[\mathbf{w}_{k}^{\dagger}\left(\mathbf{I}+c \cdot \boldsymbol{\Theta} \boldsymbol{\Theta}^{\dagger}\right) \mathbf{w}_{k}\right]$ is a strictly convex function of $\mathbf{w}_{k}$ because $\mathbf{I}+c \cdot \boldsymbol{\Theta} \Theta^{\dagger} \succ 0$ [6], so that (3) and are convex. Therefore, DOWNLINKBEAMFORMING is strictly convex and strong duality holds.

To obtain the Lagrangian of DOWNLINKBEAMFORMING, we first rewrite DOWNLINKBEAMFORMING as

$$
\begin{aligned}
\operatorname{minimize} & \sum_{\forall k \in \mathcal{K}}\left[\mathbf{w}_{k}^{\dagger}\left(\mathbf{I}+c \cdot \Theta \Theta^{\dagger}\right) \mathbf{w}_{k}\right], \\
\text { subject to } & \sum_{j \neq k}\left|\mathbf{w}_{j}^{\dagger} \mathbf{h}_{k}\right|^{2}-\frac{\left|\mathbf{w}_{k}^{\dagger} \mathbf{h}_{k}\right|^{2}}{\gamma_{k}}+\varsigma_{k} \leq 0, \forall k \in \mathcal{K}, \\
& \sum_{\forall k \in \mathcal{K}}\left(\mathbf{w}_{k}^{\dagger} \mathbf{I} \mathbf{w}_{k}\right)-P_{\max } \leq 0 .
\end{aligned}
$$


Then, the Lagrangian of DownLINKBEAMFORMING is

$$
\begin{aligned}
& L\left(\mathbf{w}_{1}, \mathbf{w}_{2}, \ldots, \mathbf{w}_{K}, \boldsymbol{\lambda}, \mu\right) \\
= & \sum_{\forall k \in \mathcal{K}}\left[\mathbf{w}_{k}^{\dagger}\left(\mathbf{I}+c \cdot \boldsymbol{\Theta} \boldsymbol{\Theta}^{\dagger}\right) \mathbf{w}_{k}\right]+\sum_{\forall k \in \mathcal{K}}\left[\lambda _ { k } \left(\sum_{j \neq k}\left|\mathbf{w}_{j}^{\dagger} \mathbf{h}_{k}\right|^{2}\right.\right. \\
& \left.\left.-\frac{\left|\mathbf{w}_{k}^{\dagger} \mathbf{h}_{k}\right|^{2}}{\gamma_{k}}+\varsigma_{k}\right)\right]+\mu\left[\sum_{\forall k \in \mathcal{K}}\left(\mathbf{w}_{k}^{\dagger} \mathbf{I} \mathbf{w}_{k}\right)-P_{\max }\right] \\
= & \sum_{\forall k \in \mathcal{K}}\left\{\mathbf { w } _ { k } ^ { \dagger } \left[\mu \mathbf{I}+\mathbf{I}+c \cdot \boldsymbol{\Theta} \Theta^{\dagger}+\sum_{j \neq k}\left(\lambda_{j} \mathbf{h}_{j} \mathbf{h}_{j}^{\dagger}\right)\right.\right. \\
& \left.\left.-\frac{\lambda_{k}}{\gamma_{k}} \mathbf{h}_{k} \mathbf{h}_{k}^{\dagger}\right] \mathbf{w}_{k}\right\}+\sum_{\forall k \in \mathcal{K}}\left(\lambda_{k} \varsigma_{k}\right)-\mu P_{\max },
\end{aligned}
$$

where $\lambda_{k}$ is the Lagrange multiplier for the $k$-th constraint in (7), $\lambda$ contains all the Lagrange multipliers for the constraints in (7), and $\mu$ is the Lagrangian multiplier for (8). The Lagrangian dual function of DOWNLINKBEAMFORMING is:

$$
\begin{aligned}
g(\boldsymbol{\lambda}, \mu)= & \inf _{\forall \mathbf{w}_{k}} L\left(\mathbf{w}_{1}, \mathbf{w}_{2}, \ldots, \mathbf{w}_{K}, \boldsymbol{\lambda}, \mu\right) \\
= & \inf _{\forall \mathbf{w}_{k}}\left\{\sum _ { \forall k \in \mathcal { K } } \left\{\mathbf { w } _ { k } ^ { \dagger } \left[(\mu+1) \mathbf{I}+c \cdot \boldsymbol{\Theta} \boldsymbol{\Theta}^{\dagger}+\sum_{j \neq k}\left(\lambda_{j} \mathbf{h}_{j} \mathbf{h}_{j}^{\dagger}\right)\right.\right.\right. \\
& \left.\left.\left.-\frac{\lambda_{k}}{\gamma_{k}} \mathbf{h}_{k} \mathbf{h}_{k}^{\dagger}\right] \mathbf{w}_{k}\right\}\right\}+\sum_{\forall k \in \mathcal{K}}\left(\lambda_{k} \varsigma_{k}\right)-\mu P_{\max }
\end{aligned}
$$

The Lagrangian dual problem of DOWNLINKBEAMFORMING is therefore given as:

\section{DOWNLINKBEAMFORMINGDUAL.}

$$
\begin{gathered}
\operatorname{maximize} g(\boldsymbol{\lambda}, \mu), \\
\text { subject to } \lambda_{k} \geq 0, \forall k \in \mathcal{K}, \\
\mu \geq 0 .
\end{gathered}
$$

Because there is no constraint on $\left\{\mathbf{w}_{k} \mid k \in \mathcal{K}\right\}$, in order to prevent $g(\boldsymbol{\lambda})$ from being minus infinity, we must have:

$(\mu+1) \mathbf{I}+c \cdot \boldsymbol{\Theta} \Theta^{\dagger}+\sum_{j \neq k}\left(\lambda_{j} \mathbf{h}_{j} \mathbf{h}_{j}^{\dagger}\right)-\frac{\lambda_{k}}{\gamma_{k}} \mathbf{h}_{k} \mathbf{h}_{k}^{\dagger} \succeq 0, \forall k \in \mathcal{K}$.

Therefore, DownLINKBEAMFORMINGDUAL is equivalent to the following:

$$
\begin{aligned}
\operatorname{maximize} & \sum_{\forall k \in \mathcal{K}}\left(\lambda_{k} \varsigma_{k}\right)-\mu P_{\max }, \\
\text { subject to } & (\mu+1) \mathbf{I}+c \cdot \boldsymbol{\Theta} \Theta^{\dagger}+\sum_{j \neq k}\left(\lambda_{j} \mathbf{h}_{j} \mathbf{h}_{j}^{\dagger}\right) \\
& -\frac{\lambda_{k}}{\gamma_{k}} \mathbf{h}_{k} \mathbf{h}_{k}^{\dagger} \succeq 0, \forall k \in \mathcal{K}, \\
& \lambda_{k} \geq 0, \forall k \in \mathcal{K}, \\
& \mu \geq 0 .
\end{aligned}
$$

The following theorem shows that DownLINKBEAMFORMING is equivalent to an uplink beamforming problem.

Theorem 2. The problem DOWNLINKBEAMFORMINGDUAL is equivalent to the following optimization problem:

\section{UPLINKBEAMFORMING.}

minimize $\sum_{\forall k \in \mathcal{K}} \hat{P}_{k}$

subject to $\frac{\hat{P}_{k} \mathbf{v}_{k}^{\dagger} \mathbf{h}_{k} \mathbf{h}_{k}^{\dagger} \mathbf{v}_{k}}{\mathbf{v}_{k}^{\dagger}\left[\sum_{j \neq k}\left(\hat{P}_{j} \mathbf{h}_{j} \mathbf{h}_{j}^{\dagger}\right)+\boldsymbol{\Sigma}\right] \mathbf{v}_{k}} \geq \gamma_{k}, \forall k \in \mathcal{K}$.

The problem UPLINKBEAMFORMING is a virtual uplink beamforming problem for the same tier-2 BS and tier-2 users in DOWNLINKBEAMFORMING, where $\hat{P}_{k}$ is the uplink transmission power of the $k$-th tier-2 user, $\mathbf{v}_{k}$ is the uplink beamformer for the $k$-th tier-2 user, and $\boldsymbol{\Sigma}=(\mu+1) \mathbf{I}+c \cdot \Theta \Theta^{\dagger}$ is the noise covariance matrix at the tier-2 BS antennas. Moreover, $\hat{P}_{k}$ is equivalent to $\lambda_{k}$ for $k \in \mathcal{K}$.

The proof of Theorem 2 is similar to the proof of Theorem 1 in [5] and hence omitted.

Theorem 1 and Theorem 2 allow us to solve DownLINKBEAMFORMING by solving UPLINKBEAMFORMING, where the way of solving UPLINKBEAMFORMING is well known. In UPLINKBEAMFORMING, the optimal uplink beamformer of user $k$ can be obtained as:

$$
\mathbf{v}_{k}^{*}=\frac{\left[\sum_{j \neq k}\left(\hat{P}_{j}^{*} \mathbf{h}_{j} \mathbf{h}_{j}^{\dagger}\right)+\boldsymbol{\Sigma}^{*}\right]^{-1} \mathbf{h}_{k}}{\mathbf{h}_{k}^{\dagger}\left[\sum_{j \neq k}\left(\hat{P}_{j}^{*} \mathbf{h}_{j} \mathbf{h}_{j}^{\dagger}\right)+\boldsymbol{\Sigma}^{*}\right]^{-1} \mathbf{h}_{k}},
$$

where $\hat{P}_{k}^{*}=\lambda_{k}^{*}$ for $k \in \mathcal{K}$ and $\boldsymbol{\Sigma}^{*}=\left(\mu^{*}+1\right) \mathbf{I}+c \cdot \boldsymbol{\Theta} \boldsymbol{\Theta}^{\dagger}$. The optimal values of $\lambda_{k}$ for $k \in \mathcal{K}$ and $\mu$ can be found by solving DownLINKBEAMFORMINGDUAL. The problem DOWNLINKBEAMFORMINGDUAL is a semi-definite programming (SDP) which can be solved by CVX [7]. The optimal downlink beamformer for user $k$ in DOWNLINKBEAMFORMING can be calculated as [5]

$$
\mathbf{w}_{k}^{*}=\varepsilon_{k} \mathbf{v}_{k}^{*},
$$

where $\left[\begin{array}{llll}\varepsilon_{1}^{2} & \varepsilon_{2}^{2} & \cdots & \varepsilon_{K}^{2}\end{array}\right]^{T}=\boldsymbol{\Omega}^{-1}\left[\begin{array}{llll}\varsigma_{1} & \varsigma_{2} & \cdots & \varsigma_{K}\end{array}\right]^{T}, \boldsymbol{\Omega}(k, k)=$ $\frac{\left|\left(\mathbf{v}_{k}^{*}\right)^{\dagger} \mathbf{h}_{k}\right|^{2}}{\gamma_{k}}$ and $\boldsymbol{\Omega}(j, k)=-\left|\left(\mathbf{v}_{j}^{*}\right)^{\dagger} \mathbf{h}_{k}\right|^{2}$ for $j \neq k$. One useful observation from (22) is that the optimal uplink beamformers gives the directions of the optimal downlink beamformers [8].

\section{IMPACT OF POWER BudGeT}

In this section, we study the behavior of the tier-2 BS when its power budget $P_{\max }$ varies. Clearly, when an instance of DOWNLINKBEAMFORMING is feasible, there exists a value $P_{\text {feasible }}$ such that DownLINKBEAMForming is only feasible when $P_{\max } \geq P_{\text {feasible. The following lemma states that when }}$ the tier-2 BS saturates its power at the optimal solution of DownLINKBEAMFORMING, then the tier-2 BS will also use all its power when the power budget is reduced.

Lemma 1. Suppose for a given instance of DownLINKBEAMFORMING, the tier-2 BS uses power level $P_{\max }$ at the optimal point of DownLINKBEAMFORMING. Also, suppose $\delta>0$ and $P_{\max }-\delta \geq P_{\text {feasible. Then, for the same }}$ instance of DOWNLINKBEAMFORMING with power budget changed to $P_{\max }-\delta$, the tier- $2 B S$ will also use all the power. 
Proof. If at the optimal point of DownLINKBEAMFORMING with power budget $P_{\max }-\delta$, the tier-2 BS uses power $P<$ $P_{\max }-\delta$, then it should also use $P$ when the power budget is $P_{\max }$, which contradicts with the assumption that the tier-2 BS uses power level $P_{\max }$ when the power budget is $P_{\max }$.

Based on Lemma 1, we can predict the change of the interference power from the tier-2 BS to the tier-1 BS when the tier-2 BS saturates its power and the power budget reduces.

Theorem 3. Suppose $P_{\max }>P_{\text {feasible, }} \delta>0$, and $P_{\max }-$

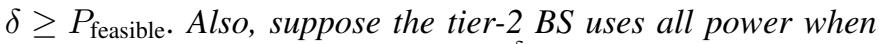
its power budget is $P_{\max }$. Let $\left\{\mathbf{w}_{k}^{-\delta, *} \mid k \in \mathcal{K}\right\}$ and $\left\{\mathbf{w}_{k}^{*} \mid k \in\right.$ $\mathcal{K}\}$ be the sets of optimal downlink beamformers of the same instance of DOWNLINKBEAMFORMING when the tier-2 BS has a power budget of $P_{\max }-\delta$ and $P_{\max }$, respectively. Then

$$
\sum_{\forall k \in \mathcal{K}}\left\|\left(\mathbf{w}_{k}^{-\delta, *}\right)^{\dagger} \boldsymbol{\Theta}\right\|^{2}>\sum_{\forall k \in \mathcal{K}}\left\|\left(\mathbf{w}_{k}^{*}\right)^{\dagger} \boldsymbol{\Theta}\right\|^{2} \text {. }
$$

Proof. Define $f^{*}(P)$ as the optimal value of the objective function of the problem DownLINKBEAMFORMING, where $P$ specifies the power budget of the tier-2 BS, i.e., $\sum_{\forall k \in \mathcal{K}}\left\|\mathbf{w}_{k}\right\|^{2} \leq P$. Then, because of Theorem 1, we have [6, Section 5.6.2]:

$$
f^{*}\left(P_{\max }-\delta\right) \geq f^{*}\left(P_{\max }\right)-\mu^{*}(-\delta) .
$$

where $\mu^{*}$ is the optimal Lagrangian multiplier of (8) when the tier-2 BS has a power budget of $P_{\max }$. Note that $\mu^{*}>0$ because of Theorem 1 and complementary slackness. Therefore, we can conclude from (24) that

$$
f^{*}\left(P_{\max }-\delta\right)>f^{*}\left(P_{\max }\right)
$$

From Lemma 1 we know that $\sum_{\forall k \in \mathcal{K}}\left\|\mathbf{w}_{k}^{-\delta, *}\right\|^{2}=P_{\max }-$ $\delta$. And from the assumption of the theorem statement $\sum_{\forall k \in \mathcal{K}}\left\|\mathbf{w}_{k}^{*}\right\|^{2}=P_{\max }$. Then,

$$
\begin{aligned}
& f^{*}\left(P_{\max }-\delta\right)>f^{*}\left(P_{\max }\right) \\
\Rightarrow & \sum_{\forall k \in \mathcal{K}}\left\|\mathbf{w}_{k}^{-\delta, *}\right\|^{2}+c \cdot \sum_{\forall k \in \mathcal{K}}\left\|\left(\mathbf{w}_{k}^{-\delta, *}\right)^{\dagger} \boldsymbol{\Theta}\right\|^{2} \\
& >\sum_{\forall k \in \mathcal{K}}\left\|\mathbf{w}_{k}^{*}\right\|^{2}+c \cdot \sum_{\forall k \in \mathcal{K}}\left\|\left(\mathbf{w}_{k}^{*}\right)^{\dagger} \boldsymbol{\Theta}\right\|^{2} \\
\Rightarrow & P_{\max }-\delta+c \cdot \sum_{\forall k \in \mathcal{K}}\left\|\left(\mathbf{w}_{k}^{-\delta, *}\right)^{\dagger} \boldsymbol{\Theta}\right\|^{2} \\
& >P_{\max }+c \cdot \sum_{\forall k \in \mathcal{K}}\left\|\left(\mathbf{w}_{k}^{*}\right)^{\dagger} \boldsymbol{\Theta}\right\|^{2} \\
\Rightarrow & \sum_{\forall k \in \mathcal{K}}\left\|\left(\mathbf{w}_{k}^{-\delta, *}\right)^{\dagger} \boldsymbol{\Theta}\right\|^{2}-\frac{\delta}{c}>\sum_{\forall k \in \mathcal{K}}\left\|\left(\mathbf{w}_{k}^{*}\right)^{\dagger} \boldsymbol{\Theta}\right\|^{2} \\
\Rightarrow & \sum_{\forall k \in \mathcal{K}}\left\|\left(\mathbf{w}_{k}^{-\delta, *}\right)^{\dagger} \boldsymbol{\Theta}\right\|^{2}>\sum_{\forall k \in \mathcal{K}}\left\|\left(\mathbf{w}_{k}^{*}\right)^{\dagger} \boldsymbol{\Theta}\right\|^{2} .
\end{aligned}
$$

Theorem 3 has the following implication: Suppose at an instance of DOWNLINKBEAMFORMING with power budget $P_{\max }$, the tier-2 BS has to use all the power, then the tier2 BS will cause more interference to the tier-1 BS if $P_{\max }$ is reduced (assuming feasibility holds). This phenomenon can also be explained intuitively: If the tier- 2 BS always operates at its maximum allowed power level, then as the power budget reduces towards $P_{\text {feasible, }}$, the optimal downlink beamformers should go towards those when $c=0$, which means that the tier-2 BS cares less about paying cost to the tier-1 BS because it has to satisfy its downlink users' target SINRs first.

\section{EFFECT OF CHARGING INTERFERENCE}

From (21) and (22), we can see that the tier-1 BS can change the behavior of the tier-2 BS by adjusting $c$, because $c$ determines the optimal uplink beamformers $\left\{\mathbf{v}_{k}^{*} \mid k \in \mathcal{K}\right\}$ which determine the directions of the optimal downlink beamformers of the tier-2 BS. We are interested in analysing what will happen to the power consumption of the tier-2 BS and the interference power from the tier- 2 BS to the tier- $1 \mathrm{BS}$ if $c$ varies. The analysis is involved because we cannot obtain a closed-form solution of the optimal downlink beamformers of the tier-2 BS in terms of $c$. On the other hand, we are able to obtain the following analytical results.

Theorem 4. Suppose DownLInKBEAMFORMING is feasible for a given instance, where $c=c_{1}$. Then for any other $c$ other than $c_{1}$, DOWNLINKBEAMFORMING is also feasible.

Proof. The optimal solution of DOWNLINKBEAMFORMING when $c=c_{1}$ is feasible for the same problem when $c=c_{2} \neq$ $c_{1}$, because all SINR targets are satisfied by using the optimal beamformers when $c=c_{1}$.

Lemma 2. Suppose DownlinkBEAMForming is feasible for a given instance, and suppose the tier-2 BS uses all its power at the optimal solutions when $c=c_{1}$ and $c=c_{2}$. Denote $\left\{\lambda_{1}^{*, c_{1}}, \lambda_{2}^{*, c_{1}}, \ldots, \lambda_{K}^{*, c_{1}}, \mu^{*, c_{1}}\right\}$ and $\left\{\lambda_{1}^{*, c_{2}}, \lambda_{2}^{*, c_{2}}, \ldots, \lambda_{K}^{*, c_{2}}, \mu^{*, c_{2}}\right\}$ as the optimal solutions of DOWNLINKBEAMFORMINGDUAL when $c=c_{1}$ and $c=c_{2}$, respectively. Then,

$$
\frac{\lambda_{k}^{*, c_{1}}}{\lambda_{k}^{*, c_{2}}}=\frac{\mu^{*, c_{1}}+1}{\mu^{*, c_{2}}+1}=\frac{c_{1}}{c_{2}} .
$$

The proof of Lemma 2 involves examining the Lagrangian dual problem of DOWNLINKBEAMFORMINGDUAL, i.e., the dual of the dual of DownLINKBEAMFORMING. The proof is omitted due to space limitations. Lemma 2 results in the following theorem.

Theorem 5. Suppose DownlinkBEAMFORMING is feasible for a given instance, and suppose the tier-2 BS uses all its power at the optimal solutions when $c=c_{1}$ and $c=c_{2}$. Then, the optimal downlink beamformers of the tier-2 BS when $c=c_{1}$ are the same as those when $c=c_{2}$.

Proof. Let $\left\{\mathbf{v}_{1}^{*, c_{1}}, \mathbf{v}_{2}^{*, c_{1}}, \ldots, \mathbf{v}_{K}^{*, c_{1}}\right\}$ and $\left\{\mathbf{v}_{1}^{*, c_{2}}, \mathbf{v}_{2}^{*, c_{2}}, \ldots, \mathbf{v}_{K}^{*, c_{2}}\right\}$ be the optimal uplink beamformers for UPLINKBEAMFORMING when $c=c_{1}$ and when $c=c_{2}$, respectively. Then because $\frac{\lambda_{k}^{*, c_{1}}}{\lambda_{k}^{*, c_{2}}}=\frac{\mu^{*, c_{1}}+1}{\mu^{*, c_{2}+1}}=\frac{c_{1}}{c_{2}}, \hat{P}_{k}^{*, c_{1}}=\lambda_{k}^{*, c_{1}}$, and $\hat{P}_{k}^{*, c_{2}}=\lambda_{k}^{*, c_{2}}$ for $k \in \mathcal{K}, \frac{\mathbf{v}_{k}^{*, c_{1}}}{\left\|\mathbf{v}_{k}^{*, c_{1}}\right\|}=\frac{\mathbf{v}_{k}^{*, c_{2}}}{\left\|\mathbf{v}_{k}^{*, c_{2}}\right\|}$ for $k \in \mathcal{K}$, where $\hat{P}_{k}^{*, c_{1}}$ is the 
optimal uplink power for user $k$ in UPLINKBEAMFORMING when $c=c_{1}$ and $\hat{P}_{k}^{*, c_{2}}$ is the optimal uplink power for user $k$ in UPLINKBEAMFORMING when $c=c_{2}$. The theorem then follows because the tier-2 BS uses all power for $c=c_{1}$ and $c=c_{2}$ and the directions of the optimal beamformers when $c=c_{1}$ are the same as those when $c=c_{2}$.

Theorem 5 implies the following: If the tier-2 BS operates at its maximum allowable power for a range of charges which is denoted by $\mathcal{C}$, then the interference power from the tier-2 BS to the tier-1 BS does not change if the tier-1 BS use any $c \in \mathcal{C}$. The tier-1 BS can then make more profit if $c_{2}>c_{1}$, where $c_{1}, c_{2} \in \mathcal{C}$.

\section{Simulation STUdies}

In the following, we perform simulation studies on two simulation settings:

S-1 $N^{\text {Tier-1 }}=1, M=3, Q_{m}=0.1$ for all $m \in \mathcal{M}$, $N^{\text {Tier-2 }}=5, K=3$, and $\sigma_{k}^{2}=1$ for all $k \in \mathcal{K}$. Note that in this setting, since $N^{\text {Tier-2 }}>N^{\text {Tier-1 }}+K$, the tier-2 BS has enough degree of freedom (DoF) to nullify its interference to the tier-1 BS.

S-2 Everything is the same as it is in S-1 except $N^{\text {Tier-1 }}=10$. Note that in this setting, since $N^{\text {Tier-2 }}<N^{\text {Tier-1 }}$, the tier$2 \mathrm{BS}$ in general does not have enough DoF to nullify its interference to the tier-1 BS.

In both of the above simulation settings, each element of all the channel links is i.i.d. complex Gaussian random variable with zero mean and unit variance. Also, all channel links remain the same in the same randomly generated network topology.

We plot three performance indicators for different values of $c$ under some randomly generated realizations of S-1 and $\mathrm{S}-2$, where the beamformers of the tier-2 BS are the optimal solution of DownlinkBEAMForming. The three performance indicators are:

1) The total transmission power of the tier- 2 BS, i.e., $\sum_{\forall k \in \mathcal{K}}\left\|\mathbf{w}_{k}^{*}\right\|^{2}$ which is denoted as "Tier-2 BS Transmission Power" in Fig. 1 and Fig. 2.

2) The interference power from the tier-2 BS to the tier-2 BS, i.e., $\sum_{\forall k \in \mathcal{K}}\left\|\left(\mathbf{w}_{k}^{*}\right)^{\dagger} \boldsymbol{\Theta}\right\|^{2}$ which is denoted as "Int. Power from Tier-2 BS to Tier-1 BS" in Fig. 1 and Fig. 2.

3) The profit of the tier-1 BS by charging the tier-2 BS for causing interfernece, i.e., $c \cdot \sum_{\forall k \in \mathcal{K}}\left\|\left(\mathbf{w}_{k}^{*}\right)^{\dagger} \boldsymbol{\Theta}\right\|^{2}$ which is denoted as "Tier-1 BS Profit" in Fig. 1 and Fig. 2.

Note that although the channel gains $\boldsymbol{\Theta},\left\{\mathbf{h}_{k} \mid k \in \mathcal{K}\right\}$, and $\left\{\boldsymbol{\vartheta}_{m, k} \mid m \in \mathcal{M}, k \in \mathcal{K}\right\}$ are randomly generated, they remain fixed for each of the following figures at different values of $c$.

Fig. 1 shows a randomly generated network topology using simulation setting S-1. We can observe that:

1) When $P_{\max }=4$, we can see that the transmission power of the tier-2 BS increases as $c$ increases, but the tier-2 BS still has more power than it needs at the maximum $c$ concerned in Fig. 1. Also, the interference power from the tier-2 BS to the tier-1 BS decreases as $c$ increases, and the tier-2 BS is able to completely avoid causing any interference at high values of $c$. This observation
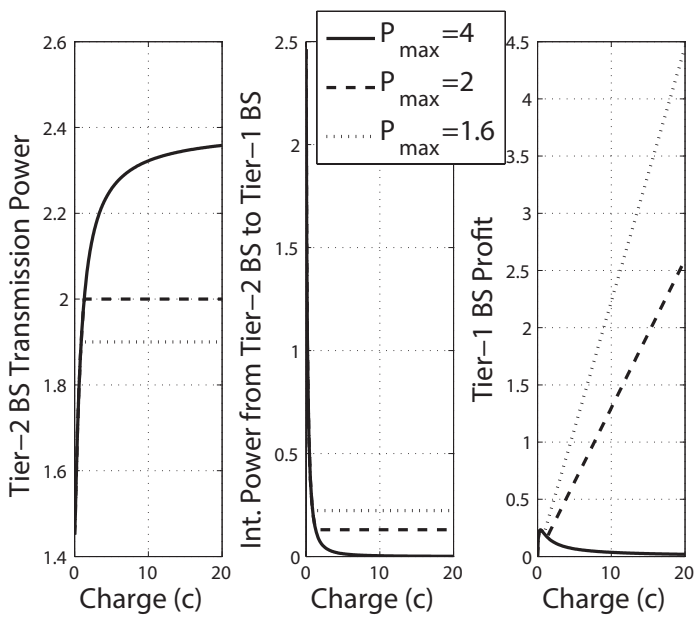

Fig. 1. A randomly generated network topology for $\mathrm{S}-1$, where $N^{\text {Tier-2 }}=5$, $K=3$, and $N^{\text {Tier-1 }}=1$. The solid lines, dashed lines, and the dotted lines show the performance when $P_{\max }=4,2$, and 1.6, respectively.

can be supported by the Tier-1 BS Profit curve, where the profit made by the tier-1 BS first increases but then decreases as $c$ is increases.

2) When $P_{\max }=2$, we can see that the tier-2 BS has to use all its power when $c$ gets large. Also, when $c$ increases, the interference power from the tier-2 BS to the tier-1 BS first decreases and then remains at a positive value. This phenomenon is supported by Theorem 5 , i.e., the optimal downlink beamformers of the tier-2 BS do not change when the charge $c$ changes among values that would cause the tier-2 BS to use all its power, so that the interference from the tier-2 BS to the tier-1 BS does not change for the same set of $c$ values. As a result, the profit made by the tier-1 BS becomes a linear function on $c$ when $c$ is large enough.

3) When $P_{\max }=1.6$, we can see that at large values of $c$, the interference caused by the tier-2 BS is larger than that when $P_{\max }=2$, which reflects the exact same phenomenon as predicted by Theorem 3 .

Fig. 2 shows a randomly generated network topology using simulation setting S-2. As seen, the tier-2 BS does not need to use all its power for the range of $c$ concerned in Fig. 2 when $P_{\max }=1$. However, the tier-2 BS is unable to completely avoid causing interference to the tier-1 BS even when $P_{\max }=1$, since the interference power from the tier-2 BS to the tier-1 BS remains at about 3.45 for large $c$ values. For the $P_{\max }=0.7$ case, the tier-2 BS needs to use all its power at large $c$ values, and the interference power from the tier-2 BS to the tier-1 BS remains at about 3.7 when $c$ becomes large. When $P_{\max }$ further reduces to 0.6 , the interference power from the tier-2 BS increases to about 4.1.

\section{Discussions on Possible Applications}

Although Fig. 1 and Fig. 2 are two special cases, the observations from the two figures are more or less the same 

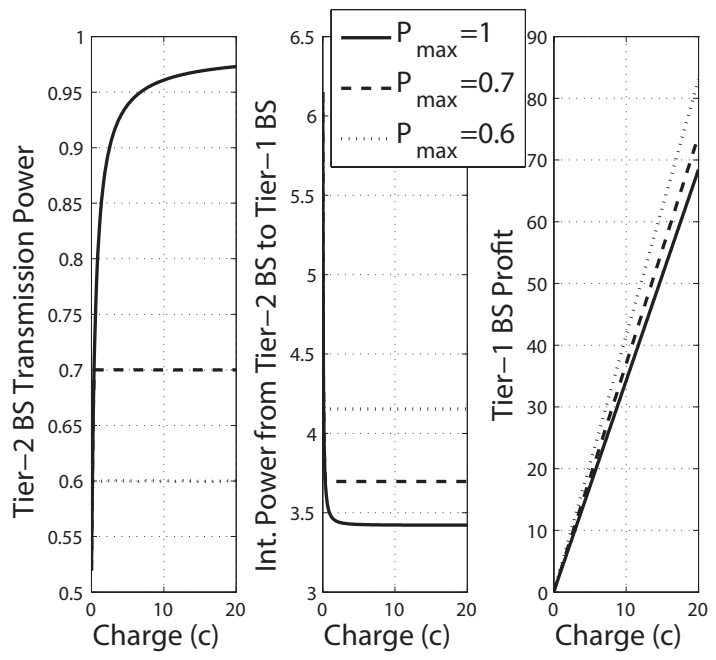

Fig. 2. A randomly generated network topology for $\mathrm{S}-2$, where $N^{\text {Tier-2 }}=5$, $K=3$, and $N^{\text {Tier- } 1}=10$. The solid lines, dashed lines, and the dotted lines show the performance when $P_{\max }=1,0.7$, and 0.6 , respectively.

as many other random realizations on the same simulation settings. We are able to summarize the findings as follows:

1) The interference from the tier- 2 BS to the tier- $1 \mathrm{BS}$ is not an increasing function of $c$.

2) When the tier-2 BS has enough power budget and enough DoF, it is capable of avoiding interference leakage to the tier-1 BS at large $c$ values. Under such scenarios, the tier-1 BS can reduce the interference from the tier-2 BS to zero by increasing $c$, but the tier-1 BS should not issue a very large $c$ if its objective is to maximize its profit.

3) On the other hand, in massive MIMO setups when the tier-1 BS has much more antennas than the tier-2 BS, the tier-2 BS would not be able to suppress all its interference (even if the tier-2 BS has infinite power budget) unless the channels between the tier-1 BS and the tier-2 BS are highly correlated, i.e., $\Theta$ is of low rank.

4) When the tier-2 BS does not have enough power budget or enough DoF, it may not be able to completely avoid causing any interference to the tier-1 BS at large $c$ values. When this happens, the tier-1 BS is certain that it can earn more profit by increasing $c$.

Based on the above observations, it is possible for the tier$1 \mathrm{BS}$ to control the interference from tier-2 BS and make profit without knowing the downlink channels of the tier2 BS. Specifically, the tier-1 BS can issue a number of $c$ values to the tier-2 $\mathrm{BS}$, let the tier-2 $\mathrm{BS}$ do the downlink beamforming optimizations, and ask the tier-2 BS to feed back the interference which would be caused to the tier-1 BS. From the feedback of the tier-2 BS, the tier-1 BS can decide to formally use one of the tested $c$ values if that $c$ value satisfies the target of interference control and/or revenue. Otherwise, the tier-1 BS can test other $c$ values based on the knowledge on how the tier- 2 BS reacts on the already tested $c$ values.
Alternatively, the tier-1 BS can issue a charge, measure the interference from the tier-2 BS, and decides whether it needs to change the charge. The actual design on determining the tier-1 BS's charge depends on the application scenario and we leave the details in future work.

\section{CONCLUSION}

Inspired by the scenarios where only limited backhaul links may be available across different tiers of BSs in HetNets, we discussed the downlink beamforming problem of a tier-2 BS, where the tier-2 BS is charged by a nearby tier-1 BS based on how much interference the tier- 2 BS causes. Theoretical analyses and simulation studies were preformed to understand the behavior of the tier- 2 BS under various settings. The observations on the behavior of the tier-2 BS suggested a simple mechanism of controlling the interference from the tier$2 \mathrm{BS}$ to the tier-1 $\mathrm{BS}$ which does not require the knowledge of the tier-2 BS's downlink channels at the tier-1 BS. The same mechanism can also be used for the tier-1 BS to make profit by charging the tier- 2 BS for causing interference. We are focusing on extending the work to networks with larger number of BSs and the application of Stackelberg game model into the design of the charge.

\section{REFERENCES}

[1] N. Bhushan, J. Li, D. Malladi, R. Gilmore, D. Brenner, A. Damnjanovic, R. Sukhavasi, C. Patel, and S. Geirhofer, "Network densification: the dominant theme for wireless evolution into 5G," IEEE Communications Magazine, vol. 52, no. 2, pp. 82-89, February 2014.

[2] L. Lu, G. Li, A. Swindlehurst, A. Ashikhmin, and R. Zhang, "An overview of massive MIMO: Benefits and challenges," IEEE Journal of Selected Topics in Signal Processing, vol. 8, no. 5, pp. 742-758, Oct 2014.

[3] K. Hosseini, J. Hoydis, S. ten Brink, and M. Debbah, "Massive MIMO and small cells: How to densify heterogeneous networks," in IEEE International Conference on Communications (ICC), June 2013, pp. 5442-5447.

[4] J. Ghimire and C. Rosenberg, "Revisiting scheduling in heterogeneous networks when the backhaul is limited," IEEE Journal on Selected Areas in Communications, vol. 33, no. 10, pp. 2039-2051, Oct 2015.

[5] W. Yu and T. Lan, "Transmitter optimization for the multiantenna downlink with per-antenna power constraints," IEEE Transactions on Signal Processing, vol. 55, no. 6, pp. 2646-2660, June 2007.

[6] S. Boyd and L. Vandenberghe, Convex Optimization. New York, NY, USA: Cambridge University Press, 2004.

[7] CVX Research, Inc., "CVX: Matlab software for disciplined convex programming, version 2.0," http://cvxr.com/cvx, Aug. 2012.

[8] D. Nguyen and T. Le-Ngoc, "Multiuser downlink beamforming in multicell wireless systems: A game theoretical approach," IEEE Transactions on Signal Processing, vol. 59, no. 7, pp. 3326-3338, July 2011. 